\title{
FRANEL INTEGRALS OF ORDER FOUR
}

\author{
RICHARD J. MCINTOSH
}

(Received 5 January 1993; revised 28 June 1993)

Communicated by J. H. Loxton

\begin{abstract}
Let $((x))=x-\lfloor x\rfloor-1 / 2$ be the sawtooth function. If $a, b, c$ and $e$ are positive integers, then the integral of $((a x))((b x))((c x))((e x))$ over the unit interval involves Apostol's generalized Dedekind sums. By expressing this integral as a lattice-point sum we obtain an elementary method for its evaluation. We also give an elementary proof of the reciprocity law for the third generalized Dedekind sum.
\end{abstract}

1991 Mathematics subject classification (Amer. Math. Soc.): 26A09, 11 F20.

\section{Introduction}

In 1924 Franel [5] gave the formula

$$
\int_{0}^{1}((a x))((b x)) d x=\frac{(a, b)^{2}}{12 a b}
$$

where $a$ and $b$ are positive integers, $(a, b)$ denotes the greatest common divisor of $a$ and $b$, and $((x))=x-\lfloor x\rfloor-1 / 2$ is the first Bernoulli periodic function. Here $\lfloor x\rfloor$ denotes the greatest integer $\leq x$. (For a proof see [7, pp. 170-171] or [9, pp. 24-25]). By symmetry about the midpoint $x=1 / 2$ it is easy to see that the corresponding integral of the product $((a x))((b x))((c x))$ is equal to 0 . This naturally leads us to the question - Is there a simple formula for

$$
\int_{0}^{1}((a x))((b x))((c x))((e x)) d x
$$

where $a, b, c$ and $e$ are positive integers?

Unfortunately, (2) does not have a simple formula, even when $c=e=1$. A general formula involves Apostol's generalized Dedekind sums [2] and related cotangent

(C) 1996 Australian Mathematical Society 0263-6115/95 \$A2.00+0.00 
sums. In this paper an elementary method is given for evaluating (2) and for special cases explicit formulae are provided.

\section{An equivalent lattice-point sum}

Denote the integral in (2) by $\mathrm{I}(a, b, c, e)$. Since

$$
((x))=-\frac{1}{\pi} \sum_{n=1}^{\infty} \frac{\sin (2 \pi n x)}{n}
$$

it follows that

$$
\begin{aligned}
\mathrm{I}(a, b, c, e) & =\frac{1}{\pi^{4}} \int_{0}^{1} \lim _{n \rightarrow \infty} \sum_{1 \leq s, t, u, v \leq n} \frac{\sin (2 \pi s a x) \sin (2 \pi t b x) \sin (2 \pi u c x) \sin (2 \pi v e x)}{s t u v} d x \\
& =\frac{1}{16 \pi^{4}} \int_{0}^{1} \lim _{n \rightarrow \infty} \sum_{\substack{1 \leq|s|,|t|,|u|,|v| \leq n\\
}} \frac{\cos \{2 \pi(s a+t b+u c+v e) x\}}{s t u v} d x \\
& =\frac{1}{16 \pi^{4}} \sum_{\substack{s, t, u, v \in \mathbb{Z}-\{0\} \\
s a+t b+u c+v e=0}} \frac{1}{\text { stuv }} .
\end{aligned}
$$

Observe that the last sum, which we will denote by $\mathrm{L}(a, b, c, e)$, is taken over all lattice points $(s, t, u, v)$ with non-zero coordinates lying on the hyperplane in $\mathbb{R}^{4}$ orthogonal to $(a, b, c, e)$. To see that $\mathrm{L}(a, b, c, e)$ is absolutely convergent, we follow an argument due to Peter Montgomery [8]. Firstly, observe that

$$
\sum_{\substack{s, t, u, v \in \mathbb{Z}-\{0\} \\ s a+t b+u c+v e=0}} \frac{1}{|s t u v|}=\sum_{M=-\infty}^{\infty} \mathrm{F}_{-M}(a, b) \mathrm{F}_{M}(c, e),
$$

where

$$
\mathrm{F}_{M}(a, b)=\sum_{\substack{s, t \in \mathbb{Z}-\{0\} \\ s a+t b=M}} \frac{1}{|s t|} .
$$

By a straight forward calculation $\mathrm{F}_{0}(a, b) \leq 2 \sum_{k=1}^{\infty} 1 / k^{2}=\pi^{2} / 3$. The proof of the absolute convergence of $\mathrm{L}(a, b, c, e)$ will be complete once we show that $\mathrm{F}_{M}(a, b)=$ $O((\log M) / M)$ as $M \rightarrow \infty$. We now consider the case $a=b=1$. The general case is similar. By partial fractions we obtain

$$
\mathrm{F}_{M}(1,1)=\sum_{k=1}^{M-1} \frac{1}{k(M-k)}+2 \sum_{k=1}^{\infty} \frac{1}{k(M+k)}
$$




$$
\begin{aligned}
& =\frac{1}{M} \sum_{k=1}^{M-1}\left(\frac{1}{k}+\frac{1}{M-k}\right)+\frac{2}{M} \sum_{k=1}^{\infty}\left(\frac{1}{k}-\frac{1}{M+k}\right) \\
& =\frac{2}{M} \sum_{k=1}^{M-1} \frac{1}{k}+\frac{2}{M} \sum_{k=1}^{M} \frac{1}{k}=O\left(\frac{\log M}{M}\right),
\end{aligned}
$$

which is what we wanted to prove.

THEOREM 1. Let $k$ be a positive integer. Then

(i) $\mathrm{L}(k a, k b, k c, k e)=\mathrm{L}(a, b, c, e)$;

(ii) $\mathrm{L}(k a, k b, k c, e)=k^{-1} \mathrm{~L}(a, b, c, e) \quad$ if $\quad(k, e)=1$;

(iii) $\mathrm{L}(a, b, c, e)=\frac{(a, b, c, e)^{4}}{(a, b, c)(a, b, e)(a, c, e)(b, c, e)} \mathrm{L}(\alpha, \beta, \gamma, \epsilon)$, where

$$
\begin{array}{ll}
\alpha=\frac{a(a, b, c, e)^{2}}{(a, b, c)(a, b, e)(a, c, e)}, & \beta=\frac{b(a, b, c, e)^{2}}{(a, b, c)(a, b, e)(b, c, e)}, \\
\gamma=\frac{c(a, b, c, e)^{2}}{(a, b, c)(a, c, e)(b, c, e)}, & \epsilon=\frac{e(a, b, c, e)^{2}}{(a, b, e)(a, c, e)(b, c, e)}
\end{array}
$$

are triplet-wise relatively prime, that is, $(\alpha, \beta, \gamma)=(\alpha, \beta, \epsilon)=(\alpha, \gamma, \epsilon)=$ $(\beta, \gamma, \epsilon)=1$.

PROOF. For (i) we have

$$
\mathrm{L}(k a, k b, k c, k e)=\sum_{\substack{s, t, u, v \in \mathbb{Z}-\{0\} \\
s k a+t k b+u k c+v k e=0}} \frac{1}{s t u v}=\sum_{\begin{array}{c}
s, t, u, v \in \mathbb{Z}-\{0\} \\
s a+t b+u c+v e=0
\end{array}} \frac{1}{s t u v}=\mathrm{L}(a, b, c, e) .
$$

For (ii) observe that if $s k a+t k b+u k c+v e=0$, then $k$ divides $v$ since $k$ is relatively prime to $e$. Setting $v=k w$, we get

$$
\mathrm{L}(k a, k b, k c, e)=\sum_{\substack{s, t, u, v \in \mathbb{Z}-\{0\} \\ s k a+t k b+u k c+v e=0}} \frac{1}{s t u v}=\frac{1}{k} \sum_{\substack{s, t, u, w \in \mathbb{Z}-\{0\} \\ s a+t b+u c+w e=0}} \frac{1}{s t u w}=\frac{1}{k} \mathrm{~L}(a, b, c, e) .
$$

For (iii) we first apply (i) to factor out $(a, b, c, e)$, and then we apply (ii) four times to factor out $(a, b, c) /(a, b, c, e),(a, b, e) /(a, b, c, e),(a, c, e) /(a, b, c, e)$, and $(b, c, e) /(a, b, c, e)$.

Numerical calculations suggest that the function $f(a, b, c, e)$ defined by

$$
f(a, b, c, e)=\frac{240 a^{3} b^{3} c^{3} e^{3}(a, b, c)(a, b, e)(a, c, e)(b, c, e)}{(a, b)^{2}(a, c)^{2}(a, e)^{2}(b, c)^{2}(b, e)^{2}(c, e)^{2}(a, b, c, e)^{4}} \mathrm{I}(a, b, c, e)
$$

is integer-valued, but a proof is out of our reach. 
REMARK. If we apply the above technique to the Franel integral of order two we get

$$
\int_{0}^{1}((a x))((b x)) d x=-\frac{1}{4 \pi^{2}} \sum_{\substack{m, n \in \mathbb{Z}-\{0\} \\ m a+n b=0}} \frac{1}{m n}=\frac{(a, b)^{2} \zeta(2)}{2 a b \pi^{2}}=\frac{(a, b)^{2}}{12 a b}
$$

\section{The evaluation of $\mathrm{L}(a, b, c, e)$}

Again, following Peter Montgomery's idea, define

$$
\mathrm{L}_{M}(a, b)=\sum_{\substack{s, t \in \mathbb{Z}-\{0\} \\ s a+t b=M}} \frac{1}{s t}
$$

and observe that

$$
\mathrm{L}(a, b, c, e)=\sum_{M=-\infty}^{\infty} \mathrm{L}_{-M}(a, b) \mathrm{L}_{M}(c, e)=\sum_{M=-\infty}^{\infty} \mathrm{L}_{M}(a, b) \mathrm{L}_{M}(c, e) .
$$

The evaluation of $\mathrm{L}_{M}(a, b)$ depends on the divisibility of $M$ by $a$ and $b$. Here $M$ is an integer and $a$ and $b$ are positive integers. Theorem 2 below considers all five possible cases. These are (i) $M=0$, (ii) both $a$ and $b$ divide $M$, (iii) only $b$ divides $M$ (if only $a$ divides $M$, then we interchange the roles of $a$ and $b$ ), (iv) both $a$ and $b$ do not divide $M$, but $(a, b)$ divides $M$, and (v) $(a, b)$ does not divide $M$. In order to simplify the expressions involved in these five cases we employ the following notation:

$$
\begin{aligned}
(a, b)=\operatorname{gcd}\{a, b\}, & {[a, b]=\operatorname{lcm}\{a, b\}, } \\
A=\frac{a}{(a, b)}, & B=\frac{b}{(a, b)}, \\
\bar{A}=A^{-1}(\bmod B), & \bar{B}=B^{-1}(\bmod A) .
\end{aligned}
$$

THEOREM 2. (i) $\mathrm{L}_{0}(a, b)=-\pi^{2} / 3 A B$.

(ii) $\mathrm{L}_{[a, b] N}(a, b)=-2 / A B N^{2}$ if $N \neq 0$.

(iii) $\mathrm{L}_{b N}(a, b)=-\frac{A}{B N^{2}}+\frac{\pi}{B N} \cot \left(\frac{N \pi}{A}\right)$ if $A \nmid N$.

(iv) $\mathrm{L}_{(a, b) N}(a, b)=\frac{\pi}{N} \cot \left(\frac{\bar{B} N \pi}{A}\right)+\frac{\pi}{N} \cot \left(\frac{\bar{A} N \pi}{B}\right)$ if $A \nmid N, B \nmid N$.

(v) $\mathrm{L}_{N}(a, b)=0$ if $(a, b) \nmid N$. 
PROOF. Since

$$
\sum_{\substack{s, t \in \mathbb{Z}-\{0\} \\ s a+t b=[a, b] N}} \frac{1}{s t}=\frac{1}{A B} \sum_{\substack{u, v \in \mathbb{Z}-\{0\} \\ u+v=N}} \frac{1}{u v}
$$

where $s=B u$ and $t=A v$, it follows that $\mathrm{L}_{[a, b] N}(a, b)=\mathrm{L}_{N}(1,1) / A B$. A straight forward calculation shows that $\mathrm{L}_{0}(1,1)=-2 \zeta(2)=-\pi^{2} / 3$, and when $N \neq 0$ we get

$$
\begin{aligned}
\mathbf{L}_{N}(1,1) & =\sum_{k=1}^{N-1} \frac{1}{k(N-k)}-2 \sum_{k=1}^{\infty} \frac{1}{k(N+k)} \\
& =\frac{1}{N} \sum_{k=1}^{N-1}\left(\frac{1}{k}+\frac{1}{N-k}\right)-\frac{2}{N} \sum_{k=1}^{\infty}\left(\frac{1}{k}-\frac{1}{N+k}\right) \\
& =-\frac{2}{N^{2}} .
\end{aligned}
$$

For (iii) we begin with $\mathrm{L}_{b N}(a, b)=\mathrm{L}_{N}(A, 1) / B$. If $A \nmid N$ then

$$
\begin{aligned}
\mathrm{L}_{N}(A, 1)=\sum_{k \in \mathbb{Z}-\{0\}} \frac{1}{k(N-A k)} & =\sum_{k=1}^{\infty}\left\{\frac{1}{k(N-A k)}-\frac{1}{k(N+A k)}\right\} \\
& =\frac{1}{N} \sum_{k=1}^{\infty}\left\{\left(\frac{1}{k}+\frac{A}{N-A k}\right)-\left(\frac{1}{k}-\frac{A}{N+A k}\right)\right\} \\
& =2 A \sum_{k=1}^{\infty} \frac{1}{N^{2}-A^{2} k^{2}} \\
& =-\frac{A}{N^{2}}+\frac{\pi}{N} \cot \left(\frac{N \pi}{A}\right),
\end{aligned}
$$

since

$$
\pi \cot (\pi z)=\frac{1}{z}+2 z \sum_{k=1}^{\infty} \frac{1}{z^{2}-k^{2}}=\sum_{k=-\infty}^{\infty} \frac{z}{z^{2}-k^{2}}, \quad z \notin Z,
$$

(see, for example, [1, p.75] or [6, p.197]).

For (iv) we have

$$
\begin{aligned}
\mathrm{L}_{(a, b) N}(a, b)=\mathrm{L}_{N}(A, B) & =\sum_{k=-\infty}^{\infty} \frac{1}{(\bar{B} N-A k)(\bar{A} N+B k)} \\
& =\frac{1}{N} \sum_{k=-\infty}^{\infty}\left(\frac{A}{\bar{B} N-A k}+\frac{B}{\bar{A} N+B k}\right)
\end{aligned}
$$




$$
=\frac{\pi}{N} \cot \left(\frac{\bar{B} N \pi}{A}\right)+\frac{\pi}{N} \cot \left(\frac{\bar{A} N \pi}{B}\right),
$$

where $A \nmid N, B \nmid N$ and $\bar{A} A+\bar{B} B=1$.

For (v) observe that if $(a, b) \nmid N$ then the sum $\mathrm{L}_{N}(a, b)$ is empty.

\section{Some special cases}

A general formula for $\mathrm{L}(a, b, c, e)$ is very cumbersome. It involves the greatest common divisors of various subsets of $\{a, b, c, e\}$. However, formulae for special cases can be obtained easily with the help of Theorem 2. For the most simple case, we have

$$
\mathrm{L}(1,1,1,1)=\sum_{M=-\infty}^{\infty} \mathrm{L}_{M}^{2}(1,1)=\mathrm{L}_{0}^{2}(1,1)+2 \sum_{M=1}^{\infty} \mathrm{L}_{M}^{2}(1,1)=\frac{\pi^{4}}{9}+8 \zeta(4)=\frac{\pi^{4}}{5},
$$

by Theorem 2(i) and (ii). A somewhat more difficult example is

$$
\begin{aligned}
\mathrm{L}(a, 1,1,1) & =\sum_{M=-\infty}^{\infty} \mathrm{L}_{M}(a, 1) \mathrm{L}_{M}(1,1) \\
& =\mathrm{L}_{0}(a, 1) \mathrm{L}_{0}(1,1)+2 \sum_{N=1}^{\infty} \mathrm{L}_{a N}(a, 1) \mathrm{L}_{a N}(1,1)+2 \sum_{\substack{N=1 \\
a \nmid N}}^{\infty} \mathrm{L}_{N}(a, 1) \mathrm{L}_{N}(1,1) \\
& =\frac{\pi^{4}}{9 a}+\frac{8 \zeta(4)}{a^{3}}-4 \sum_{\substack{N=1 \\
a \nmid N}}^{\infty} \sum_{k=1}^{\infty} \frac{2 a}{N^{2}\left(N^{2}-a^{2} k^{2}\right)} \\
& =\frac{\pi^{4}}{9 a}+\frac{4 \pi^{4}}{45 a^{3}}+\frac{8}{a} \sum_{k=1}^{\infty} \frac{1}{k^{2}} \sum_{\substack{N=1 \\
a \nmid N}}^{\infty}\left(\frac{1}{N^{2}}-\frac{1}{N^{2}-a^{2} k^{2}}\right) \\
& =\frac{\pi^{4}}{9 a}+\frac{4 \pi^{4}}{45 a^{3}}+\frac{2 \pi^{4}}{9 a}-\frac{2 \pi^{4}}{9 a^{3}}-\frac{8}{a} \sum_{k=1}^{\infty} \frac{1}{k^{2}} \sum_{\substack{N=1 \\
a \nmid N}}^{\infty} \frac{1}{N^{2}-a^{2} k^{2}} \\
& =\left\{\frac{1}{3 a}-\frac{2}{15 a^{3}}\right\} \pi^{4},
\end{aligned}
$$

where we have used (3) and the identity

$$
\sum_{\substack{N=1 \\ a \nmid N}}^{\infty} \frac{1}{N^{2}-a^{2} k^{2}}=0, \quad k \neq 0 .
$$


For the remaining cases we do not assume that $a$ and $b$ are relatively prime. In the next case we use the fact, which follows from Theorem $2(\mathrm{v})$, that $\mathrm{L}_{M}(k, k)=0$ whenever $k$ does not divide $M$. Thus, we have

$$
\begin{aligned}
\mathrm{L}(a, a, b, b) & =\sum_{M=-\infty}^{\infty} \mathrm{L}_{M}(a, a) \mathrm{L}_{M}(b, b) \\
& =\mathrm{L}_{0}(a, a) \mathrm{L}_{0}(b, b)+2 \sum_{N=1}^{\infty} \mathrm{L}_{[a, b] N}(a, a) \mathrm{L}_{[a, b] N}(b, b) \\
& =\frac{\pi^{4}}{9}+\frac{8(a, b)^{4} \zeta(4)}{a^{2} b^{2}}=\left\{\frac{1}{9}+\frac{4(a, b)^{4}}{45 a^{2} b^{2}}\right\} \pi^{4} .
\end{aligned}
$$

In the introduction we claimed that some Franel integrals of order four do not have a simple formula. By a simple formula we mean a rational function of $a, b, c, e$, and the greatest common divisors of various subsets of $\{a, b, c, e\}$. An example not having a simple formula is

$$
\begin{aligned}
\mathrm{L}(a, a, b, 1)= & \sum_{M=-\infty}^{\infty} \mathrm{L}_{M}(a, a) \mathrm{L}_{M}(1, b) \\
= & \mathrm{L}_{0}(a, a) \mathrm{L}_{0}(1, b)+2 \sum_{N=1}^{\infty} \mathrm{L}_{[a, b] N}(a, a) \mathrm{L}_{[a, b] N}(1, b) \\
& +2 \sum_{\substack{N=1 \\
B \nmid N}}^{\infty} \mathrm{L}_{a N}(a, a) \mathrm{L}_{a N}(1, b) \\
= & \frac{\pi^{4}}{9 b}+\frac{8(a, b)^{4} \zeta(4)}{a^{2} b^{3}}+\frac{4}{a} \sum_{\substack{N=1 \\
B \nmid N}}^{\infty}\left\{\frac{b}{a N^{4}}-\frac{\pi}{N^{3}} \cot \left(\frac{A N \pi}{B}\right)\right\} \\
= & \left\{\frac{1}{9 b}+\frac{2 b}{45 a^{2}}+\frac{2(a, b)^{4}}{45 a^{2} b^{3}}+\frac{16}{3 a} S_{3}(A, B)\right\} \pi^{4},
\end{aligned}
$$

where $S_{3}(h, k)$ is a generalized Dedekind sum introduced by Apostol [2] and defined by

$$
S_{3}(h, k)=\sum_{r=1}^{k-1} \frac{r}{k} B_{3}\left(\frac{h r}{k}-\left\lfloor\frac{h r}{k}\right\rfloor\right),
$$

where $h$ and $k$ are relatively prime positive integers and $B_{n}(t)$ is the $n$th Bernoulli polynomial defined by the generating function

$$
\frac{x e^{x t}}{e^{x}-1}=\sum_{n=0}^{\infty} B_{n}(t) \frac{x^{n}}{n !} .
$$


This generalized Dedekind sum can also be expressed by

$$
\begin{aligned}
S_{3}(h, k) & =\sum_{r=1}^{k-1} B_{1}\left(\frac{r}{k}-\left\lfloor\frac{r}{k}\right\rfloor\right) B_{3}\left(\frac{h r}{k}-\left\lfloor\frac{h r}{k}\right\rfloor\right) \\
& =-\frac{3}{8 k^{3}} \sum_{r=1}^{k-1} \cot \left(\frac{r h \pi}{k}\right) \cot \left(\frac{r \pi}{k}\right) \csc ^{2}\left(\frac{r \pi}{k}\right) \\
& =-\frac{3}{4 \pi^{3}} \sum_{\substack{r=1 \\
k \nmid r}}^{\infty} \frac{1}{r^{3}} \cot \left(\frac{r h \pi}{k}\right),
\end{aligned}
$$

(see, for example, [3, p.2, p.4]).

By direct calculation

$$
S_{3}(1, k)=-\frac{\left(k^{2}-1\right)\left(k^{2}-4\right)}{120 k^{3}} .
$$

In general there is no closed expression for the sum $S_{3}(h, k)$. However, $S_{3}$ enjoys the following reciprocity law.

THEOREM 3. For relatively prime positive integers $h$ and $k$,

$$
120 h k^{3} S_{3}(h, k)+120 k h^{3} S_{3}(k, h)=-h^{4}-k^{4}+5 h^{2} k^{2}-3 .
$$

ProOF. By (6) and (4) we have

$$
\begin{aligned}
& -\frac{4 \pi^{4}}{3 h^{2}} S_{3}(h, k)-\frac{4 \pi^{4}}{3 k^{2}} S_{3}(k, h)=\frac{\pi}{h^{2}} \sum_{\substack{r=1 \\
k \nmid r}}^{\infty} \frac{1}{r^{3}} \cot \left(\frac{r h \pi}{k}\right)+\frac{\pi}{k^{2}} \sum_{\substack{r=1 \\
h \nmid r}}^{\infty} \frac{1}{r^{3}} \cot \left(\frac{r k \pi}{h}\right) \\
& =\sum_{\substack{r=1 \\
k \nmid r}}^{\infty} \sum_{t=-\infty}^{\infty} \frac{h k}{h^{2} r^{2}\left(h^{2} r^{2}-k^{2} t^{2}\right)}+\sum_{\substack{r=1 \\
h \nmid r}}^{\infty} \sum_{t=-\infty}^{\infty} \frac{h k}{k^{2} r^{2}\left(k^{2} r^{2}-h^{2} t^{2}\right)} \\
& =2 \sum_{\substack{r=1 \\
k \nmid r}}^{\infty} \sum_{\substack{t=1 \\
h \nmid t}}^{\infty} \frac{h k}{h^{2} r^{2}\left(h^{2} r^{2}-k^{2} t^{2}\right)}-2 \sum_{\substack{r=1 \\
h \nmid r}}^{\infty} \sum_{\substack{t=1 \\
k \nmid t}}^{\infty} \frac{h k}{k^{2} r^{2}\left(h^{2} t^{2}-k^{2} r^{2}\right)} \\
& +\sum_{\substack{r=1 \\
k \nmid r}}^{\infty} \sum_{t=-\infty}^{\infty} \frac{h k}{h^{2} r^{2}\left(h^{2} r^{2}-h^{2} k^{2} t^{2}\right)}-\sum_{\substack{r=1 \\
h \nmid r}}^{\infty} \sum_{t=-\infty}^{\infty} \frac{h k}{k^{2} r^{2}\left(h^{2} k^{2} t^{2}-k^{2} r^{2}\right)} \\
& =2 \sum_{\substack{r=1 \\
k \nmid r}}^{\infty} \sum_{\substack{t=1 \\
h \nmid t}}^{\infty} \frac{h k}{h^{2} r^{2}\left(h^{2} r^{2}-k^{2} t^{2}\right)}-2 \sum_{\substack{t=1 \\
h \nmid t}}^{\infty} \sum_{\substack{r=1 \\
k \nmid r}}^{\infty} \frac{h k}{k^{2} t^{2}\left(h^{2} r^{2}-k^{2} t^{2}\right)} \\
& +\sum_{t=-\infty}^{\infty}\left\{\frac{k}{h^{3}} \sum_{\substack{r=1 \\
k \nmid r}}^{\infty} \frac{1}{r^{2}\left(r^{2}-k^{2} t^{2}\right)}+\frac{h}{k^{3}} \sum_{\substack{r=1 \\
h \nmid r}}^{\infty} \frac{1}{r^{2}\left(r^{2}-h^{2} t^{2}\right)}\right\}
\end{aligned}
$$




$$
\begin{aligned}
= & -2 \sum_{\substack{r=1 \\
k \nmid r}}^{\infty} \sum_{\substack{=1 \\
h \nmid t}}^{\infty} \frac{1}{h k r^{2} t^{2}}+\frac{k}{h^{3}} \sum_{\substack{r=1 \\
k \nmid r}}^{\infty} \frac{1}{r^{4}}+\frac{h}{k^{3}} \sum_{\substack{r=1 \\
h \nmid r}}^{\infty} \frac{1}{r^{4}} \\
& +2 \sum_{t=1}^{\infty}\left\{\frac{1}{k h^{3} t^{2}} \sum_{\substack{r=1 \\
k \nmid r}}^{\infty}\left(\frac{1}{r^{2}-k^{2} t^{2}}-\frac{1}{r^{2}}\right)+\frac{1}{h k^{3} t^{2}} \sum_{\substack{r=1 \\
h \nmid r}}^{\infty}\left(\frac{1}{r^{2}-h^{2} t^{2}}-\frac{1}{r^{2}}\right)\right\} \\
=- & \frac{\left(h^{2}-1\right)\left(k^{2}-1\right) \pi^{4}}{18 h^{3} k^{3}}+\frac{\left(k^{4}-1\right) \pi^{4}}{90 h^{3} k^{3}}+\frac{\left(h^{4}-1\right) \pi^{4}}{90 h^{3} k^{3}}-\frac{\left(k^{2}-1\right) \pi^{4}}{18 h^{3} k^{3}} \\
& -\frac{\left(h^{2}-1\right) \pi^{4}}{18 h^{3} k^{3}}+\frac{2}{h^{3} k^{3}} \sum_{t=1}^{\infty} \frac{1}{t^{2}}\left\{\sum_{\substack{r=1 \\
k \nmid r}}^{\infty} \frac{k^{2}}{r^{2}-k^{2} t^{2}}+\sum_{\substack{r=1 \\
h \nmid r}}^{\infty} \frac{h^{2}}{r^{2}-h^{2} t^{2}}\right\} \\
= & \frac{\pi^{4}}{90 h^{3} k^{3}}\left(h^{4}+k^{4}-5 h^{2} k^{2}+3\right),
\end{aligned}
$$

since the last term equals 0 by (5). This completes the proof of the reciprocity law.

Our next example, $\mathrm{L}(a, b, 1,1)$, can be evaluated in two different ways, and by combining the results we obtain an expression for a rather complicated cotangent sum in terms of generalized Dedekind Sums. By keeping the variables $a$ and $b$ together we obtain a formula involving two generalized Dedekind Sums. In particular, we have

$$
\begin{aligned}
\mathrm{L}(a, b, 1,1)= & \sum_{M=-\infty}^{\infty} \mathrm{L}_{M}(a, b) \mathrm{L}_{M}(1,1) \\
= & \mathrm{L}_{0}(a, b) \mathrm{L}_{0}(1,1) \\
& +2 \sum_{N=1}^{\infty} \mathrm{L}_{[a, b] N}(a, b) \mathrm{L}_{[a, b] N}(1,1)+2 \sum_{\substack{N=1 \\
A \nmid N}}^{\infty} \mathrm{L}_{b N}(a, b) \mathrm{L}_{b N}(1,1) \\
& +2 \sum_{\substack{N=1 \\
B \nmid N}}^{\infty} \mathrm{L}_{a N}(a, b) \mathrm{L}_{a N}(1,1)+2 \sum_{\substack{N=1 \\
A \nmid N}}^{\infty} \mathrm{L}_{(a, b) N}(a, b) \mathrm{L}_{(a, b) N}(1,1) \\
= & \frac{(a, b)^{2} \pi^{4}}{9 a b}+\frac{4(a, b)^{4} \pi^{4}}{45 a^{3} b^{3}}+\frac{4}{b^{2}} \sum_{\substack{N=1 \\
A \nmid N}}^{\infty}\left\{\frac{a}{b N^{4}}-\frac{\pi}{B N^{3}} \cot \left(\frac{N \pi}{A}\right)\right\} \\
& +\frac{4}{a^{2}} \sum_{\substack{N=1 \\
B \nmid N}}^{\infty}\left\{\frac{b}{a N^{4}}-\frac{\pi}{A N^{3}} \cot \left(\frac{N \pi}{B}\right)\right\} \\
& \quad-\frac{4 \pi}{(a, b)^{2}} \sum_{\substack{N=1 \\
A \nmid N \\
B \nmid N}}^{\infty}\left\{\frac{1}{N^{3}} \cot \left(\frac{\bar{B} N \pi}{A}\right)+\frac{1}{N^{3}} \cot \left(\frac{\bar{A} N \pi}{B}\right)\right\}
\end{aligned}
$$




$$
=\left\{\frac{2 a}{45 b^{3}}+\frac{2 b}{45 a^{3}}+\frac{(a, b)^{2}}{9 a b}+\frac{16}{3(a, b)^{2}}\left\{S_{3}(\bar{B}, A)+S_{3}(\bar{A}, B)\right\}\right\} \pi^{4},
$$

where we have used (7) and the identity

$$
\sum_{\substack{N=1 \\ A \nmid N \\ B \nmid N}}^{\infty} \frac{1}{N^{3}} \cot \left(\frac{\bar{B} N \pi}{A}\right)=-\frac{4 \pi^{3}}{3} S_{3}(\bar{B}, A)+\frac{4 \pi^{3}}{3 B^{3}} S_{3}(1, A),
$$

which follows from $(6)$ and the fact that $\bar{B} B \equiv 1(\bmod A)$.

In the second method we begin by separating the variables $a$ and $b$ and eventually obtain a formula involving a single cotangent sum. We have

$$
\begin{aligned}
& \mathrm{L}(a, b, 1,1)=\sum_{M=-\infty}^{\infty} \mathrm{L}_{M}(a, 1) \mathrm{L}_{M}(1, b) \\
& =\mathrm{L}_{0}(a, 1) \mathrm{L}_{0}(1, b) \\
& +2 \sum_{N=1}^{\infty} \mathrm{L}_{[a, b] N}(a, 1) \mathrm{L}_{[a, b] N}(1, b)+2 \sum_{\substack{N=1 \\
A \nmid N}}^{\infty} \mathrm{L}_{b N}(a, 1) \mathrm{L}_{b N}(1, b) \\
& +2 \sum_{\substack{N=1 \\
B \nmid N}}^{\infty} \mathrm{L}_{a N}(a, 1) \mathrm{L}_{a N}(1, b)+2 \sum_{\substack{N=1 \\
a \nmid N \\
b \nmid N}}^{\infty} \mathrm{L}_{N}(a, 1) \mathrm{L}_{N}(1, b) \\
& =\frac{\pi^{4}}{9 a b}+\frac{4(a, b)^{4} \pi^{4}}{45 a^{3} b^{3}}+\frac{4}{b^{2}} \sum_{\substack{N=1 \\
A \nmid N}}^{\infty}\left\{\frac{a}{b N^{4}}-\frac{\pi}{N^{3}} \cot \left(\frac{B N \pi}{A}\right)\right\} \\
& +\frac{4}{a^{2}} \sum_{\substack{N=1 \\
B \nmid N}}^{\infty}\left\{\frac{b}{a N^{4}}-\frac{\pi}{N^{3}} \cot \left(\frac{A N \pi}{B}\right)\right\} \\
& +2 \sum_{\substack{N=1 \\
a \nmid N \\
b \nmid N}}^{\infty}\left\{\frac{a}{N^{2}}-\frac{\pi}{N} \cot \left(\frac{N \pi}{a}\right)\right\}\left\{\frac{b}{N^{2}}-\frac{\pi}{N} \cot \left(\frac{N \pi}{b}\right)\right\} \\
& =\frac{\pi^{4}}{9 a b}+\frac{a b \pi^{4}}{45}+\frac{b \pi^{4}}{45 a^{3}}+\frac{a \pi^{4}}{45 b^{3}}+\frac{(a, b)^{4} \pi^{4}}{45 a^{3} b^{3}} \\
& +\frac{8 b \pi^{4}}{3} S_{3}(1, a)+\frac{8 a \pi^{4}}{3} S_{3}(1, b)+\frac{8 \pi^{4}}{3 b^{2}} S_{3}(B, A)+\frac{8 \pi^{4}}{3 a^{2}} S_{3}(A, B) \\
& +2 \pi^{2} \sum_{\substack{N=1 \\
a \nmid N \\
b \nmid N}}^{\infty} \frac{1}{N^{2}} \cot \left(\frac{N \pi}{a}\right) \cot \left(\frac{N \pi}{b}\right)
\end{aligned}
$$




$$
\begin{aligned}
=\{ & \left.-\frac{a b}{45}+\frac{b}{9 a}+\frac{a}{9 b}+\frac{2}{9 a b}-\frac{4 b}{45 a^{3}}-\frac{4 a}{45 b^{3}}-\frac{2(a, b)^{4}}{45 a^{3} b^{3}}\right\} \pi^{4} \\
& +\frac{\pi^{4}}{a^{2} b^{2}} \sum_{\substack{r=1 \\
a \nmid r \\
b \nmid r}}^{a b-1} \cot \left(\frac{r \pi}{a}\right) \cot \left(\frac{r \pi}{b}\right) \csc ^{2}\left(\frac{r \pi}{a b}\right),
\end{aligned}
$$

where we have used (7), (8) and the identity

$$
\sum_{\substack{N=1 \\ a \nmid M \\ b \nmid N}}^{\infty} \frac{1}{N^{3}} \cot \left(\frac{N \pi}{a}\right)=-\frac{4 \pi^{3}}{3} S_{3}(1, a)+\frac{4 \pi^{3}}{3 b^{3}} S_{3}(B, A) .
$$

Combining (9) and (10) we obtain the formula

$$
\begin{aligned}
& \sum_{\substack{r=1 \\
a \neq r \\
b \nmid r}}^{a b-1} \cot \left(\frac{r \pi}{a}\right) \cot \left(\frac{r \pi}{b}\right) \csc ^{2}\left(\frac{r \pi}{a b}\right) \\
& =\frac{a^{3} b^{3}}{45}+\frac{2 a^{3}}{15 b}+\frac{2 b^{3}}{15 a}-\frac{a^{3} b}{9}-\frac{a b^{3}}{9}-\frac{2 a b}{9}+\frac{a b(a, b)^{2}}{9} \\
& +\frac{2(a, b)^{4}}{45 a b}+\frac{16 a^{2} b^{2}}{3(a, b)^{2}}\left\{S_{3}(\bar{B}, A)+S_{3}(\bar{A}, B)\right\} .
\end{aligned}
$$

\section{Concluding remarks}

Bruce Berndt gives two generalizations of (1) in [4, Lemmas 4.2 and 7.1].

Subsequent to the writing of this article, it was brought to the author's attention that George Greaves, Richard Hall, Martin Huxley and Julie Wilson are also working on Franel Integrals and have had a paper accepted by Mathematika.

\section{Acknowledgement}

Support by the Natural Sciences and Engineering Research Council of Canada is gratefully acknowledged.

\section{References}

[1] M. Abramowitz and I. E. Stegun, Handbook of mathematical functions, National Bureau of Standards Applied Mathematics Series 55 (National Bureau of Standards, Washington D.C., 1972). 
[2] T. M. Apostol, 'Generalized Dedekind sums and the transformation formulae of certain Lambert series', Duke Math. J. 17 (1950), 147-157.

[3] _ - 'Theorems on generalized Dedekind sums', Pacific J. Math. 2 (1952), 1-9.

[4] B. C. Berndt, 'Reciprocity theorems for Dedekind sums and generalizations', Adv. Math. 23 (1977), 285-316.

[5] J. Franel, 'Les suites de Farey et le problème des nombres premiers', Göttingen Nachrichten (1924), 198-201.

[6] A. S. B. Holland, Complex function theory (Elsevier, New York, 1980).

[7] E. Landau, Vorlesungen über Zahlentheorie, zweiter Band (Chelsea, New York, 1947).

[8] P. Montgomery, private correspondence, 1991.

[9] H. Rademacher and E. Grosswald, Dedekind sums, Carus Math. Monographs 16 (Math. Assoc. Amer., Washington D.C., 1972).

Department of Mathematics and Statistics

University of Regina

Regina, Saskatchewan

Canada S4S 0A2

e-mail: mcintosh@ninja.math.uregina.ca 